\title{
Clostridium difficile: a formidable foe
}

\author{
Vivian G. Loo, Michael D. Libman, Mark A. Miller, Anne-Marie Bourgault, Charles H. Frenette, \\ Mirabelle Kelly, Sophie Michaud, Tuyen Nguyen, Louise Poirier, Anne Vibien, Ruth Horn, \\ Pierre J. Laflamme, Pierre René
}

ß See related articles pages 19, 27, 33, 45 and 51

I $\mathrm{n}$ the world of infectious diseases, the confidence of the 1960s and 1970s has given way to a stark realization that our mastery of the microbial world is not absolute - as the emergence of SARS and of avian influenza has illustrated. The current outbreak of Clostridium difficile associated diarrhea (CDAD) in Montréal and other areas of Quebec is a harsh reminder that even well-known bacteria can change their behaviour and become emerging threats.

In this issue, Susan Poutanen and Andrew Simor provide a detailed review of the epidemiology, pathogenesis, clinical presentation, diagnosis, treatment and control of CDAD. ${ }^{1}$ Despite this knowledge, the incidence of CDAD continues to increase in many hospitals across Canada, the United States and elsewhere. The US Centers for Disease Control and Prevention have analyzed secular trends in the incidence of CDAD and reported a steady increase from 1987 to $2001 .^{2}$ Of 440 infectious disease physicians in the US who participated in a recent Web-based poll, 30\% reported that they are seeing higher rates of $\mathrm{CDAD}$, more severe fulminant $\mathrm{CDAD}$ and more relapsing CDAD than in the past.

During the past 18 to 24 months, many health care institutions in Montréal and other regions of Quebec have experienced a rise in the CDAD incidence (mean 28.2 per 1000 admissions, range $12.8-45.0$ per 1000 admissions), which is about 4 to 5 times the rate of 2 years ago and 5 times the national average in 1997. ${ }^{3}$ There is an overall impression that there has been an increase in the proportion of CDAD cases with severe and fatal complications and an increase in the relapse rate among affected patients. A 1997 Canadian survey ${ }^{4}$ indicated that the attributable case-fatality rate to be $1.5 \%$, and other authors have reported an attributable mortality of 0.8 to $2 \%$ for nosocomial CDAD. ${ }^{5,6}$ Five months ago, an ad hoc group formed by several Quebec medical microbiologists was established in light of concern about the rising CDAD incidence and its complication rates: this is now called the CDAD Clinical Study Investigators (CDAD-CSI) group. The group has established 4 priorities: (1) to establish the true incidence and serious complication rate of CDAD in affected participating Quebec hospitals by using standardized definitions and methods; (2) to begin in vitro studies of the bacteria in affected hospitals to determine whether increased virulence factors are present; (3) to establish urgent research protocols for therapy; and (4) to devise newer ultra-rapid methods of diagnosis.

The CDAD-CSI group has generated several hypotheses that may explain the current multicentre outbreak, as follows:

- A new virulent strain may have been introduced by im- portation or by mutation. Pulsed-field gel electrophoresis (PFGE) typing of 89 isolates from one Montréal hospital showed that $85 \%$ of the isolates are clonal in origin. A total of 18 isolates from 3 other hospitals have been typed, of which $9(50 \%)$ were found to have the identical PFGE profile. PFGE will also be performed on additional isolates from the remaining participating hospitals to determine if this dominant clone is prevalent across Montréal and other Quebec areas. Transmission of this dominant strain could occur by movement of colonized patients, or perhaps from health care providers working at multiple institutions. This dominant strain will be further analyzed by Dr. Tom Louie at the University of Calgary to determine if it is a hyperproducer of toxin and whether certain antibiotics trigger the release of toxin in large quantities.

- The hospital population has increasing proportions of immunocompromised, debilitated and elderly patients, thereby increasing the number of susceptible hosts.

- Antibiotics are the main precipitants of disease in patients colonized with C. difficile. Some data suggest that predominant use of "high-risk" antibiotics (with respect to inducing C. difficile toxin production) in hospitals leads to increased overall rates, and that global changes in hospital antibiotic utilization to "low-risk" antibiotics may abrogate nosocomial epidemics (M.A.M.: unpublished data). In some participating hospitals the outbreak followed the introduction of 8-methoxy-quinolones into the hospital formulary. These agents have increased anaerobic activity compared with older quinolones, possibly leading to more disruption of normal bowel flora.

- In many institutions, housekeeping staff has been reduced while nursing workloads have increased. C. difficile is particularly difficult to eradicate from surfaces and equipment. Compliance with hand hygiene has been shown to decrease as workloads increase. ${ }^{7}$ Decreased compliance with isolation protocols along with the increased environmental spore burden could have a synergistic effect in promoting $C$. difficile cross-infection.

- The current facilities in many hospitals are antiquated and contain few single or isolation rooms. Wards and emergency departments have become more crowded, and bed turnover is rapid. This makes containment of $C$. difficile exceedingly difficult, especially among patients with fecal incontinence. Sharing of toilet facilities between patients in multi-bed rooms is still occurring.

- A new hypothesis explored by Dial and colleagues ${ }^{8}$ in this 
issue concerns the role of proton pump inhibitors (PPIs) in promoting CDAD. Other factors that elevate the risk of CDAD include older age, recent gastrointestinal surgery and immunocompromising therapy. The use of PPIs may augment these risks in such patients.

Many infection control measures have been implemented, including contact precautions, dedicated equipment for infected patients when possible, disposable thermometers, early empirical treatment of suspected cases, infection prevention and control education for personnel, an emphasis on hand hygiene, intensified housekeeping in high-incidence units, sporicidal agents for environmental disinfection, review and potential restriction of antibiotics, and decreased turnaround time for $C$. difficile test results.

Despite these measures, no hospital in our group has been able to bring the CDAD rate to its pre-epidemic rate. Why have we not been able to break the cycle of transmission? Several obstacles hamper control measures. In an outbreak situation, spore burdens are high and require increasing intensity of environmental disinfection. In most hospitals, the housekeeping staff was already working at full capacity before the outbreak. Overburdened health care workers have had difficulty coping with the increased numbers of patients in isolation. Inadequate numbers of single rooms with dedicated bathroom facilities also facilitate transmission. Let us remember that other infectious patients also require vigilant isolation, such as those with MRSA, VRE, measles, chicken pox and tuberculosis, to name a few. The paucity of adequate handwashing facilities further compounds the problem. Improved methods to monitor antibiotic utilization are required. Taken together, this highlights the lack of surge capacity to fully combat the outbreak.

A number of unanswered questions remain. If this current strain is different, where has it come from? Why do some infected patients have serious complications, while other seemingly similar patients experience a relatively benign course? How can we prevent the occurrence of severe complications?
The answers to these questions would improve our understanding of this disease and its control. It is only through a concerted and coordinated approach, coupled with increased resources and funding, that expeditious answers will be found to the current dilemma and patient safety will be assured.

The authors are members of the Clostridium difficile Associated Diarrhea Clinical Study Investigators Group. Their affiliations appear in an online appendix (available at www.cmaj.ca/cgi/content/full/171/1/47/DC1)

Competing interests: None declared.

Contributors: Vivian Loo initiated and coordinated the commentary and, with Michael Libman and Mark Miller, drafted the commentary. All authors provided data included in the commentary, revised the draft critically for important intellectual content and approved the final version.

\section{References}

1. Poutanen SM, Simor AE. Clostridium difficile-associated diarrhea in adults [review]. CMA7 2004;171(1):51-8.

2. Archibald LK, Banerjee SN, Jarvis WR. Secular trends in hospital-acquired Clostridium difficile disease in the United States, 1987-2001. F Infect Dis 2004; 189:1585-9.

3. Hyland M, Ofner-Agostini M, Miller M, Paton S, Gourdeau M, Ishak M Nosocomial Clostridium difficile-associated diarrhea in Canada: results of the Canadian Nosocomial Infection Surveillance Program (CNISP). 1997 NCDAD prevalence Surveillance Project. Can 7 Infect Dis 2001;12(2):81-8.

4. Miller MA, Hyland M, Ofner-Agostini M, Gourdeau M, Ishak M. Morbidity, mortality, and healthcare burden of nosocomial Clostridium difficile-associated diarrhea in Canadian hospitals. Infect Control Hosp Epidemiol 2002;23:137-40.

5. Olson MM, Shanholtzer CJ, Lee JT, Gerding DN. Ten years of prospective Clostridium difficile-associated disease surveillance and treatment at the Minneapolis VA Medical Center. Infect Control Hosp Epidemiol 1994;15:371-81.

6. Prendergast TM, Marini CP, D'Angelo AJ, Sher ME, Cohen JR. Surgical patients with pseudomembranous colitis: factors affecting prognosis. Surgery 1994;116:768-74.

7. Pittet D, Mourouga P, Perneger TV. Compliance with handwashing in a teaching hospital. Infection Control Program. Ann Intern Med 1999;130(2):126-30.

8. Dial S, Alrasadi K, Manoukian C, Huang A, Menzies D. Risk of Clostridium difficile diarrhea among hospital inpatients prescribed proton pump inhibitors: cohort and case-control studies. CMA7 2004;171(1):33-8.

Correspondence to: Dr. Vivian G. Loo, Infection Control Service, Hôpital général de Montréal, D16.168-1650 ave. Cedar, Montréal QC H3G 1A4; fax 514 934-8427; vivian.loo@muhc.mcgill.ca

\section{Glycemic self-monitoring and insurance coverage}

\section{Michele Heisler}

ß S See related article page 39

A growing body of research is calling attention to significant rates of medication underuse and to adverse health consequences associated with high out-ofpocket drug costs, especially among patients with low incomes, multiple chronic illnesses or no prescription drug coverage. ${ }^{1,2}$ Studies in the United States and Canada have shown that public- and private-sector policies limiting drug coverage lead to fewer prescriptions being filled, ${ }^{3,4}$ higher rates of nursing home admissions ${ }^{5}$ and increased use of acute care services. ${ }^{3}$ Yet physicians often fail to identify patients who are burdened by high out-of-pocket expenses. ${ }^{6}$ Many patients with chronic illnesses who report having underused medications because of cost never discuss this underuse with their physicians. ${ }^{7,8}$

In this issue Samantha Bowker and colleagues ${ }^{9}$ (see page 39) contribute to this body of research and expand the policy debate on the scope of health insurance coverage into the realm of medical supplies. They examine cross-sectional baseline survey and laboratory data from 405 non-insulin-using patients with type 2 diabetes who are enrolled in a randomized controlled trial designed to assess the effect of providing free blood glucose testing supplies on glycemic control. Of these patients, $41 \%$ had at least some level of insurance coverage for blood 